\title{
Isolation and in silico Characterization of Disease Resistance Gene Analogues (RGAs) from Minor Millets
}

\author{
Subramanian Rajesh ${ }^{1,2} *$, Sivaraj Preetha ${ }^{1,2}$, Gurusamy Anand ${ }^{1}$, \\ Balasubramanian Selvi $^{1,3}$ and Navaneethakrishnan Shunmugavalli ${ }^{1}$
}

${ }^{1}$ Department of Plant Breeding and Genetics, Tamil Nadu Agricultural University (TNAU), Agricultural College and Research Institute, Killikulam, Vallanad -628252, Tamil Nadu, India

${ }^{2}$ Department of Plant Biotechnology, CPMB\&B, TNAU,

Coimbatore 641 003, Tamil Nadu, India

${ }^{3}$ Centre for Plant Breeding and Genetics, TNAU, Coimbatore, Tamil Nadu, India

*Corresponding author

\section{A B S T R A C T}

Minor millets are under-exploited plant species often dubbed as 'Crops for the future' as they are best adapted to less fertile area and can survive limited water situations and

\section{Keywords}

Setaria, Eleusine,

NBS, LRR,

Resistant gene, in silico

Article Info

Accepted:

17 December 2018

Available Online:

10 January 2019 relatively free from pests and diseases. Degenerate oligo-PCR strategy was employed to isolate resistant gene analogues from minor millets by amplifying samples using NBSLRR gene specific primers. Genomic DNA was isolated from the young leaves of minor millets viz., Foxtail millet (Setaria italica) var. CO 5 and Finger millet (Eleusine corocana) var. CO (Ra) 14 by following CTAB method with minor modifications. PCR amplification of the genomic DNA with degenerate primers, revealed presence of 500bp product in the minor millet samples typical to the size of other reported R-gene analogues. The amplicons TENAIKKM1 sequenced was of size 527 bases and RagiKKM1 was of size 509 bases, the sequences with high similarity to NBS-LRR family are deposited in GenBank, NCBI. In silico characterization of the sequences were done using bioinformatics tools by similarity search, pattern recognition and diversity analysis, which resulted in classifying the genes as those belonging to NBS-LRR class as that of other grass family NBS-LRR kind of proteins. Further, in silico characterization ensured that they belong to Non-TIR family of $\mathrm{R}$ genes as evident by the conserved residues in the kinase 1 and kinase 2 domains. The diversity among the novel RGAs characterized in this study may lead to mining of $R$ - genes in other under-exploited minor millets.

\section{Introduction}

Many plant defense responses are initiated by resistance genes, providing a mechanism by which the plant can recognize a pathogen and execute a defense against it. In recent years many different disease-resistance genes ( $\mathrm{R}$ genes) have been cloned from plants. R-genes contain a leucine-rich repeat region (LRR), a strong candidate for pathogen recognition specificity. Some of the LRR R-genes contain domains possibly involved in signal 
transduction such as nucleotide binding sites (NBS), leucine zippers, or Toll-Interleukin-1 Cytoplasmic receptor (TIR) domains. Still others contain protein kinase domains and conserved domains of unknown function (Meyers et al., 1999).

Genes from the NBS-LRR classes were reported to confer resistance to bacteria, fungi, virus, nematodes and aphids (TimmermanVaughan et al., 2000, Dogimont et al., 2014). In the R-genes, NBS region is thought to be important for ATP binding and overall functionality of the resistant protein coded by it (Saraste et al., 1990). The NBS and NBSLRR class of $R$ genes have been cloned and well characterized from Arabidopsis thaliana, flax, tobacco, tomato, rice,peanut, Kiwifruit and other under-exploited plant species (Bent et al., 1994; Mindrinos et al., 1994; Whitham et al., 1994; Grant et al., 1995; Lawrence et al., 1995; Milligan et al., 1998; Yoshimura et al., 1998; Tirumalaiandi et al., 2008, Maiti et al., 2011, Lozano et al., 2015, Li et al., 2016, Chong et al., 2017). CC-NBS-LRR class of gene from Haynaldia villosa was cloned and characterized (Xing et al., 2018).

Discovering specific genes from the gene pool can be achieved by various strategies, among them degenerate-oligo Polymerase chain reaction (PCR) is more sensitive and easily employed in isolating conserved gene sequences and would therefore be more effective in isolating potential NBS/LRR disease resistance genes (Shen et al., 1998). Using this strategy, it has been possible to amplify analogous sequences from many plant species (Kanazin et al., 1996; Leister et al., 1996; Yu et al., 1996). Leister et al., (1996) used PCR and oligonucleotide primers designed from conserved domains of $N$ (tobacco) and RPS2 (Arabidopsis) to amplify products with NBS homology to known resistance genes. Minor millets, also described as nutritious millets have received far less research and development attention than other crops with regard to crop improvement and utilization. On positive note, these crops possess inherent mechanism to resist biotic stresses and are less prone to major diseases. In the present study, attempts were made to isolate $\mathrm{R}$ genes from minor millets viz., finger millet (Eleusine coracana) and foxtail or Italian millet (Setaria italica) and characterize them using publicly available bioinformatics tools.

\section{Materials and Methods}

Seeds of minor millets, Foxtail millet (Setaria italica) var. CO 5 and Finger millet (Eleusine corocana) var. CO (Ra) 14 were obtained from the Department of Millets, Tamil Nadu Agricultural University, Coimbatore. Seeds were raised in pots and leaf samples were collected from the plants for total DNA isolation.

\section{Genomic DNA isolation}

Genomic DNA was isolated from the young leaves of finger millet (Eleusine coracana) and foxtail millet (Setaria italica) by adopting CTAB procedure (Doyle and Doyle, 1990) with necessary modifications. The leaves were subjected to pretreatment with absolute alcohol for 30 min., air dried and ground using liquid nitrogen in a prechilled Pestle and Mortar. Cells were lysed with DNA extraction buffer using liquid nitrogen. After cell lysis, the genomic DNA was separated using 6 volumes of Chloroform: isoamylalcohol (24:1) and precipitated using ice cold iso-propanol and the DNA was further purified by washing with $70 \%$ ethanol, the samples were electrophoresed in $1 \%$ Agarose gel electrophoresis for checking the quality.

\section{Primer Designing}

Oligonucleotide primers with optimized primer parameters were designed using Primer3 (Untergasser et al., 2012) based on 
the conserved domains of $N$ (tobacco) and RPS2 (Arabidopsis) genes and was to be used for amplification of products with NBS homology to known resistance genes.

\section{R-gene isolation by degenerate oligo-PCR}

PCR amplifications were carried out in a $50 \mu \mathrm{l}$ reaction containing $100 \mathrm{ng}$ genomic DNA, 0.2 $\mu M$ of each primer The primer sequences are ACK1F-5'-GGTGGGGTTGGGAAGACAAC G-3' and ACK1R-5'-CAACGCTAGTGG CAATCC-3' (designed from consensus sequences of NBS region), $0.25 \mathrm{~m} M$ each of the four dNTPs (Fermentas Life Sciences, Canada), $2 \mathrm{mM} \mathrm{MgCl} 2,1$ x Taq buffer (GeNei, India) and $2.5 \mathrm{U}$ Taq DNA polymerase (GeNei, India). PCR was performed in GeneAmp PCR6700 thermal cycler (Applied Biosystems Inc., USA) with initial denaturation at $92^{\circ} \mathrm{C}$ for $5 \mathrm{~min}$, then cycling for 30 times $92^{\circ} \mathrm{C}$ for $1 \mathrm{~min}, 53^{\circ} \mathrm{C}$ for $1 \mathrm{~min} ., 72^{\circ} \mathrm{C}$ for $1 \mathrm{~min}$, then final extension at $72^{\circ} \mathrm{C}$ for $5 \mathrm{~min}$. Amplified PCR products were electrophoresed in $1.5 \% \mathrm{w} / \mathrm{v}$ agarose gels (Xcelris Genomics, India) stained with ethidium bromide. PCR fragments were excised under UV transilluminator (Biorad, USA) and purified using GenElute ${ }^{\mathrm{TM}}$ Gel Extraction Kit (Sigma Aldrich, USA). To further confirm, the PCR products were reamplified using same PCR conditions. Amplicons of 500bp were gel eluted and subjected to sequencing in an automated ABI 3100 Genetic Analyser based on Sangers's sequencing method. The sequences are deposited in GenBank, NCBI, USA with accession numbers KM454474 and KM454475.

\section{In silico characterization and diversity analysis}

The nucleotide sequences of the cloned fragments were translated in to amino acid sequences using ExPASy (Expert Protein
Analysis System) Translate Tool, a proteomics server of Bioinformatics (www.expasy.org). The deduced amino acid sequences were subjected to motif analyses using the online version of CLUSTALW multiple alignment program (Thompson et al., 1997) of European Bioinformatics Institute (www.ebi.ac.uk). The amino acid sequences of the RGAs were compared with protein sequences deposited in the GenBank using BLASTP algorithm (Altschul et al., 1997). Pair wise comparison of RGA sequences with known $R$-gene sequences at NBS region was made. In addition to these, amino acid sequences of representative classes of RGAs isolated from the model legumes Glycine max (Kanazin et al., 1996), $\mathrm{N}$ gene of tobacco, RPS2, RPS5 and RPP8 of A. thaliana, L6 of Linum and $X A 1$ of rice were retrieved from the GenBank and were trimmed to start and end at the Ploop and GLPL motifs to facilitate accurate alignments with the sequences in the present study. Postsctipt pattern observed using ESPript 2.2 (Gouet et al., 1999). The deduced amino acid sequences of the RGAs were compared with protein sequences using WUBLAST. The alignment file output was fed to phylogenetic tree prediction tool- TREE TOP (Yushmanov and Chumakov, 1988) with boot strapping in a BLOSUM62 matrix. Best possible open reading frames were identified and pattern recognition was done using PROSITE tool in the same ExPASy server. Conserved domain (CD) search was performed in NCBI-CD database (MarchlerBauer et al., 2007) for classifying the protein to the respective protein super family.

\section{Results and Discussion}

\section{PCR amplification of Resistance Gene Analogues}

Use of PCR based strategies has been successful and has opened up an avenue for isolation and cloning of $\mathrm{R}$ genes from plants 
based on conserved motifs among the NBSLRR class resistance genes. This oligo Primed-PCR approach provides an alternative to the classical methods of transposan tagging and map-based cloning strategies (Seah et al., 1998). Earlier reports indicates coamplification of non-specific fragments apart from the expected amplicon (500-600 bp) in crops like soybean (Yu et al., 1996), rice (Mago et al., 1999) and sorghum (Totad et al., 2005). In the present study we could successfully amplify the targeted RGA fragment (expected size from the distance between the sequence motifs in $N$ gene of tobacco and RPS2 of Arabidopsis thaliana) from the genomic DNA of Eleusine coracana and Setaria italica due to high stringency PCR conditions with degenerate primers (Fig. 1). The amplicon from Setaria italica was designated as TenaiKKM1 and of Eleusine coracana as RagiKKM1. The sequences are deposited in GenBank of National Center for Biotechnology Information, USA and accessioned as KM454474 (TenaiKKM1) and KM454475 (RagiKKM1).

\section{Sequence analysis for conserved motifs}

The deduced amino acid sequences of RGAs in the present investigation were compared with other known $R$ genes viz., $N$ gene of tobacco, RPS2, RPS5 and RPP8 of A.thaliana, $X A 1$ of rice and $L 6$ of flax in NBS region using CLUSTALW multiple alignment program (Fig. 2). The various motifs of NBS, i.e., kinase-1a, kinase- 2 and kinase- $3 \mathrm{a}$ were conserved in Eleusine coracana (RAGIKKM1) and Setaria italica (TENAIKKMI) RGAs. The hydrophobic region represented by the GLPL domain was also observed in all the four RGA sequences. The Best ORF in the 5'3' reading frame of TENAIKKM was with 173 aa and for RAGIKKM1 was with 175aa which contained GGFGKTT - P Loop, LIVLDD Kinase 2 Loop and GLPL - motif. Conserved domain search identified NB-ARC (nucleotide-binding adaptor shared by APAF1 , $\mathrm{R}$ proteins, and CED-4) domain) and classified the protein to Pfam 00931 family. Pattern recognition done using PROSITE tool detected a major Protein kinase $\mathrm{C}$ phosphorylation site in both the cases. Sequence similarity search using WU-BLAST indicated good homology of the TENAIKKM1 sequences with the NBS-LRR class of proteins belonging to other cereals and grasses viz., Zea mays, Sorghum bicolor, Saccharum sp., Oryza sativa, Hordeum vulgare etc., Similar results were obtained with the RAGIKKM1 sequences that's showed $>55 \%$ homology with for the reported RGA sequences from Sorghum bicolor, Brachypodium distachianum, Triticum, Hordeum vulgare, Secale strictum and Oryza sativa. High sequence homology of the RGAs with NBS motifs of N, RPS2, RPS5, RPP8, L6 and XA1 clearly establishes that the RGAs cloned in the present investigation might belong to NBS-LRR class of $R$-genes.

Among R genes, the NBS-LRR class of genes are usually grouped into two subfamilies (Meyers et al., 1999; Pan et al., 2000) in which the subfamily I contains the TIR element (Toll-Interleukin-1 Receptor like domain) mostly present only in dicots, while subfamily II lacks the TIR domain found in both the monocots and dicots. The partial sequence of the NBS portion is usually sufficient to assign a given gene to either subfamily I or II. The last residue of the kinase-2 domain can be used to predict with 95\% accuracy whether an RGA would belongs to the TIR-NBS or the non-TIR-NBS family; conservation of tryptophan (W) at this location is tightly linked to non-TIR $R$-genes (RPS2, RPS5 and RPP8 of A. thaliana), whereas conservation of aspartic acid (D) or its uncharged derivative aspartate $(\mathrm{N})$ is characteristic of TIR class of $R$-genes ( $N$ and L6) (Meyers et al., 1999; Pan et al., 2000; Jeong et al., 2001; Penuela et al., 2002). 
Fig.1 PCR amplification of targeted R genes with degenerate primers

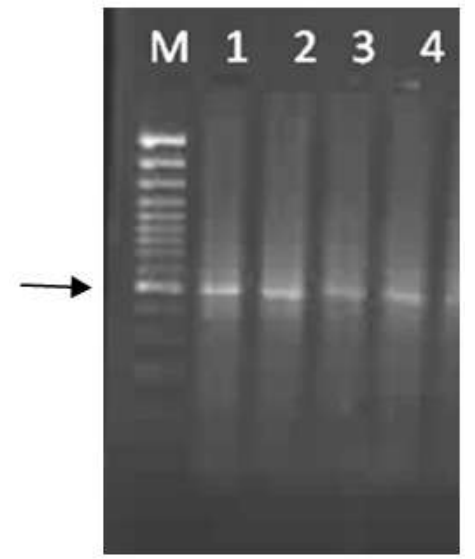

M-1 Kb DNA ladder, 1,2-foxtail millet RGA ; 3,4-finger millet RGA. Arrow indicates 500bp amplicons, size expected for NBS coding regions of R-Genes

Fig.2 Multiple sequence alignment of the novel RGAs with representative NBS-LRR class of Rgenes
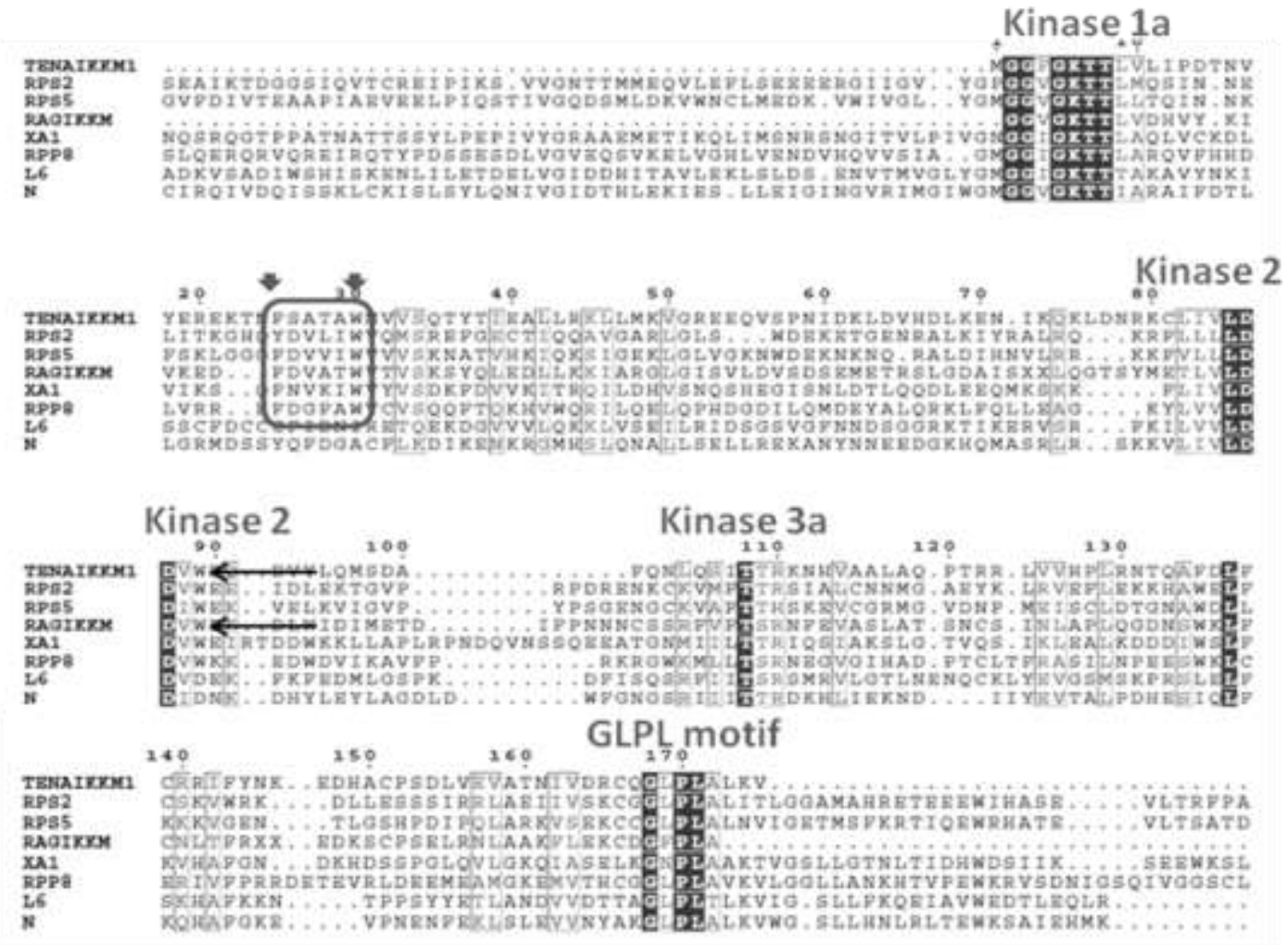

$N$ gene of tobacco, RPS2, RPS5 and RPP8 of A. thaliana, L6 of Linum and XA1 of rice in NBS region using CLUSTALW2 and Postscript pattern observed using ESPript 2.2 
Fig.3 Average distance tree for identified RGAs with known R-genes using TREETOP tool

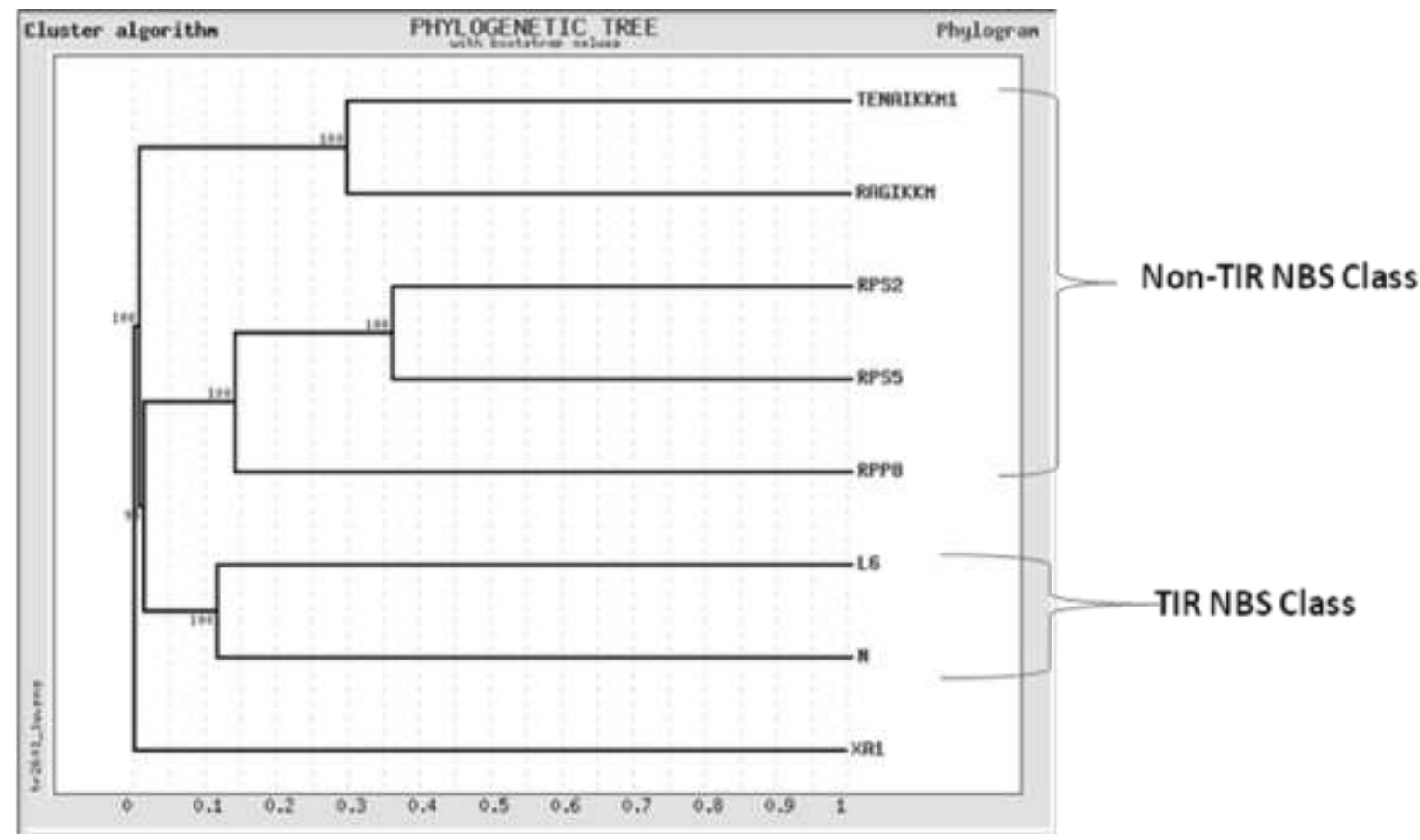

Values are boot strapped. R genes, N (acc. no.U15605), RPS2 (acc. no. U14158), RPS5 (acc. no. AF074916), RPP8 (acc. no. AF089710), XA1 (acc. no. AB002266) and L6 (acc. no. U27081)

In this study, the TENAIKKM1 and RagiKKM1 genes have Tryptophan conserved in the kinase2 loop, establishing the fact that it's a NON-TIR Class of R gene. In TIRNBS-LRR group, a characteristic consensus motif FXXXXF and a highly conserved glycine is present between kinase $1 \mathrm{a}$ and kinase-2 domains, whereas the non- TIR-NBS-LRR group contains the consensus sequence FXXXXW (Pan et al., 2000). The presence of consensus motif FXXXXW between kinase 1a and kinase-2 domains of TENAIKKM1 (KM454474) and RAGIKKM1 (KM454475) suggests RGA sequences further ensures their position in non-TIR-NBS-LRR subfamily.

\section{Diversity analysis based on phylogeny}

Phylogenetic analysis of RGA sequences with already characterized $R$-genes was done by constructing average distance tree using
BLOSUM62 in the alignment editor. TIRNBS-LRR genes evolved after the divergence of monocotyledonous and dicotyledonous crop species and clustering of RGAs occurs due to their origin from common evolutionary mechanisms. The RGAs of Eleusine coracana and Setaria italic were clustered together with well- characterized non-TIR-NBS-LRR genes RPS2, RPS5 and RPP8 of A. thaliana, while the TIR-NBS-LRR genes $N$ and $L$ were grouped as a separate cluster. The RGAs isolated from Eleusine coracana and Setaria italica were clustered together in a single subclade and exhibit their sequence similarity (Fig. 3).

Resistance gene analogues generally are putative disease resistance genes identified on the basis of their structure. Hence studies on R-genes and RGAs are still explorative in nature. Further information on R-gene sequences are necessary to delineate more 
structural domains, which would be the basis for the finding of novel RGAs in any crop plant (Totad et al., 2005). The diversity among the novel RGAs characterized in this study may open up scope and further lead to mining of R-genes in other under-exploited minor millets. To know their exact function they need to be characterized and linked with the genes actually conferring resistance phenotype. Mapping of RGAs generates perfect markers that are linked to known resistance genes. This can be employed to follow resistance genes in segregating populations, to possibly link them and then ultimately use as candidates for gene isolation.

\section{Acknowledgements}

The authors gratefully acknowledge the crews of NCBI, USA; EMBL-EBI, UK and ExPASy proteomics server of SIB, Switzerland for making the computational biology data/tools publicly available for use in analysis.

\section{References}

Altschul, S.F., Madden T.L, Schaffer, A.A., Zhang, J., Zhang, Z., Miller .W and Lipman. D.J. 1997. Gapped BLAST and PSI-BLAST: a new generation of protein database search programs. Nucleic Acids Res. 25: 3389-402.

Bent, A.F., Kundel, B.N., Dahlbeck, D., Brown, K.L., Schmidt, R., Giraudat J., Leung, J. and Staskawicz. B.J. 1994. RPS2 of Arabidopsis thaliana: a leucine-rich repeat class of plant disease resistance genes. Science 265:18561860

Chong, Z., Chen, H., Cai, T., Deng, Y., Zhuang, R., Zhang, N., Zeng, Y., Zheng, Y, Tang, R., Pan, R and Zhuang, W. 2017. Overexpression of a novel peanut NBS-LRR gene AhRRS5 enhances disease resistance to Ralstonia solanacearum in tobacco. Plant Biotechnology Journal. 15(1): 39-55

Doyle, J.J. and Doyle, J.L. 1990. Isolation of plant DNA from fresh tissue. Focus 12:13-15.

Gouet, P., Courcelle, E., Stuart, D.I. and Metoz. F. 1999. ESPript: multiple sequence alignments in PostScript. Bioinformatics. 15 305-8

Grant, M.R., Godiard, L., Straube, E., Ashfield, T and Lewald. J. 1995. Structure of the Arabidopsis RPM1 gene enabling dual specificity disease resistance. Science 269:843-46

Jeong, S.C., Hayes, A.J., Biyashev, R.M. and Saghai Maroof, M.A. 2001. Diversity and evolution of a non-TIR-NBS sequence family that clusters to a chromosomal 'hotspot' for disease resistance genes in soybean. Theoretical and Applied Genetics. 103(2-3): 406414.

Kanazin, V., Marek, L. F and Shoemaker, R.C. 1996. Resistance gene analogs are conserved and clustered in soybean. Proc. Natl. Acad. Sci. USA. 93: 1174611750

Lawrence, G.J., Flnnegan, E.J., Ayliffe, M.A., and Ellls, J.G. 1995. The L6 gene for flax rust resistance is related to the Arabidopsis bacterial resistance gene RPS2 and the tobacco viral resistance gene N. Plant Cell. 7: 1195-1206

Leister, D., Ballvora, A., Salamini, F and Gebhardt C. 1996. A PCR-based approach for isolating pathogen resistance genes from potato with potential for wide application in plants. Nature Genet. 14: 421-429

Li, Y., Zhong, Y., Huang, K. and Cheng, Z. 2016. Genome wide analysis of NBSencoding genes in kiwi fruit (Actinidia chinensis). Journal of Genetics, 95(4):997-1001

Mago, R., Nair, S. and Mohan, M. 1999. Resistance gene analogues from rice: 
cloning, sequencing and mapping. Theoretical and Applied Genetics. 99 (1-2): 50-57.

Marchler-Bauer, J. B., Anderson, M. K., Derbyshire, C., DeWeese-Scott, N. R., Gonzales, M., Gwadz, L., Hao, S., He, Hurwitz, D. I. and Jackson. J.D. 2007. CDD: a conserved domain database for interactive domain family analysis. Nucleic Acids Res., 35(suppl.1): D237D240.

Meyers, B. C., Dickerman, A. W., Michelmore, R. W., Sivaramakrishnan,S.,

Sobral, B. W and N. D. Young. 1999. Plant disease resistance genes encode members of an ancient and diverse protein family within the nucleotidebinding superfamily. Plant J. 20: $317-$ 332.

Milligan, S.B., Bodeau, J., Yaghoobi, J., Kaloshian, I., Zabel, P. and Williamson, V.A. 1998. The root knot nematode resistance gene $M i$ from tomato is a member of the leucine zipper, nucleotide binding, leucine-rich repeat family of plant genes. Plant Cell 10 (8): 1307-1319.

Mindrinos, M., Katagiri, F., Yu, G.L. and Ausubel. F.M. 1994. The Arabidopsis thaliana disease resistance gene RPS2 encodes a protein containing a nucleotide-binding site and leucine-rich repeats. Cell 78: 1089-1099.

Pan, Q., Wendel, J. and Fluhr, R. 2000. Divergent evolution of plant NBS-LRR resistance gene homologues in dicot and cereal genomes. J. Mol. Evol. 50:20313

Penuela, S., Danesh, D. and Young, N.D. 2002. Targeted isolation, sequence analysis, and physical mapping of nonTIR NBS-LRR genes in soybean. Theoretical and Applied Genetics 104 (2-3): 261-272.

Rozen, S and Skaletsky, H.J. 1998. Primer3
WWW primer tool. http://www.genome.wi.mit.edu/genome _software/other/primer3.html

Saraste, M., Sibbad, P.R. and Wittinghofer, A.1990. The P-loop-a common motif in ATP- and GTP-binding proteins. Trends in Biochemical Sciences 15(11): 430434.

Seah, S., Sivasithamparam, K.; Karakousis, A. and Lagudah, E.S. 1998. Cloning and characterization of a family of disease resistance gene analogs from wheat and barley. Theoretical and Applied Genetics 97 (5-6): 937-945.

Shen, K.A. Meyers, B.C.; Islam-Faridi, M.N., Chin, D.; Stelly, D.M. and Michelmore, R.W. 1998. Resistance gene candidates identified by PCR with degenerate oligonucleotide primers map to clusters of resistance genes in lettuce. Molecular Plant-Microbe Interaction 11(8): 815823.

Thirumalaiandi, R., Selvaraj, MG., Rajasekaran, $\mathrm{R}$ and Subbarayalu. $\mathrm{M}$. 2008. Cloning and characterization of resistance gene analogs from underexploited plant species. Electronic Journal of Biotechnology. 11(4): 1-11.

Thompson, J.D., Gibson, T.J., Plewniak, F., Jeanmougin, F. and Higgins, D.G. 1997. The ClustalX windows interface: Flexible strategies for multiple sequence alignment aided by quality analysis tools. Nucleic Acids Research 25 (24): 4876-4882.

Timmerman-Vaughan, G.M., Frew, T.J. and Weeden, N.F. 2000. Characterization and linkage mapping of R-gene analogous DNA sequences in pea (Pisum sativum L.). Theoretical and Applied Genetics 101(1-2): 241-247.

Totad, A.S., Fakrudin, B. and Kuruvinashetti, M.S. 2005. Isolation and characterization of resistance gene analogs (RGAs) from sorghum (Sorghum bicolor L. Moench). 
Euphytica 143 (1-2): 179-188.

Untergasser, A., Cutcutache, I., Koressaar, T., Ye, J., Faircloth, B.C., Remm, M and Rozen, S.G. 2012. Primer3-new capabilities and interfaces. Nucleic Acids Res. 40(15):e115

Whitham, S., Dinesh-Kumar, S.P., Choi, D., Hehl, R., Corr, C., and Baker, B. 1994. The product of the tobacco mosaic virus resistance gene N: Similarity to Toll and the interleukin-1 receptor. Cell 78: 1101-1115.

Xing, L., Hu, P., Liu, J., Witek, K., Zhou, S., Xu, J., Zhou, W., Gao, W., Huang, Z., Zhang, R., Wang X., Chen, P., Wang H., Jones, JDG., Karafia'tova', M., Vra'na, J., Bartos, J., Dolezel, J., Tian, Y., Wu, Y and Cao, A. 2018. Pm21 from Haynaldia villosa encodes a CCNBS-LRR Protein Conferring Powdery Mildew Resistance in Wheat. Molecular
Plant. 2018. 11(6): 874-878.

Yoshimura, S., Yamanouchi, U., Katayose, Y., Toki, S., Wang, Z.X., Kono, I., Kurata, N.. Yano, M., Iwata, N. and Sasaki, T. 1998. Expression of Xal a bacterial blight-resistance gene in rice is induced by bacterial inoculation. Proc. Natl. Acad. Sci. USA, 95 (4): 16631668.

Yu, Y.G., Buss, G.R., Saghai-Maroof, M.A. 1996. Isolation of a superfamily of candidate disease-resistance genes in soybean based on a conserved nucleotide- binding site. Proc. Natl. Acad. Sci. USA, 93:11751-11756.

Yushmanov S.V. and Chumakov K.M. 1988. Algorithms of the maximum topological similarity phylogenetic trees construction, Mol. Genet. Microbiol. Virol. 3: 9-15.

\section{How to cite this article:}

Subramanian Rajesh, Sivaraj Preetha, Gurusamy Anand, Balasubramanian Selvi and Navaneethakrishnan Shunmugavalli. 2019. Isolation and in silico Characterization of Disease Resistance Gene Analogues (RGAs) from Minor Millets. Int.J.Curr.Microbiol.App.Sci. 8(01): 2773-2781. doi: https://doi.org/10.20546/ijcmas.2019.801.292 